\title{
Modeling nanoscale gas sensors under realistic conditions: Computational screening of metal-doped carbon nanotubes
}

\author{
J. M. García-Lastra, ${ }^{1,2}$ D. J. Mowbray, ${ }^{1,2}$ K. S. Thygesen, ${ }^{2}$ A. Rubio, ${ }^{1,3}$ and K. W. Jacobsen ${ }^{2}$ \\ ${ }^{1}$ Nano-Bio Spectroscopy Group and ETSF Scientific Development Centre, Dpto. Física de Materiales, Universidad del País Vasco, \\ Avenue Tolosa 72, E-20018 San Sebastián, Spain \\ ${ }^{2}$ Center for Atomic-Scale Materials Design, Department of Physics, Technical University of Denmark, \\ DK-2800 Kongens Lyngby, Denmark \\ ${ }^{3}$ Fritz-Haber-Institut der Max-Planck-Gesellschaft, Berlin, Germany
}

(Received 31 May 2010; published 22 June 2010)

\begin{abstract}
We use computational screening to systematically investigate the use of transition-metal-doped carbon nanotubes for chemical-gas sensing. For a set of relevant target molecules $\left(\mathrm{CO}, \mathrm{NH}_{3}\right.$, and $\left.\mathrm{H}_{2} \mathrm{~S}\right)$ and the main components of air $\left(\mathrm{N}_{2}, \mathrm{O}_{2}\right.$, and $\left.\mathrm{H}_{2} \mathrm{O}\right)$, we calculate the binding energy and change in conductance upon adsorption on a metal atom occupying a vacancy of a $(6,6)$ carbon nanotube. Based on these descriptors, we identify the most promising dopant candidates for detection of a given target molecule. From the fractional coverage of the metal sites in thermal equilibrium with air, we estimate the change in the nanotube resistance per doping site as a function of the target molecule concentration assuming charge transport in the diffusive regime. Our analysis points to Ni-doped nanotubes as candidates for $\mathrm{CO}$ sensors working under typical atmospheric conditions.
\end{abstract}

DOI: 10.1103/PhysRevB.81.245429

PACS number(s): 73.63.-b, 68.43.-h, 73.50.Lw

\section{INTRODUCTION}

The ability to detect small concentrations of specific chemical species is fundamental for a variety of industrial and scientific processes as well as for medical applications and environmental monitoring. ${ }^{1}$ In general, nanostructured materials should be well suited for sensor applications because of their large surface to volume ratio which makes them sensitive to molecular adsorption. Specifically, carbon nanotubes (CNTs) (Ref. 2) have been shown to work remarkably well as detectors of small gas molecules. This has been demonstrated both for individual CNTs (Refs. 3-8) as well as for CNT networks. ${ }^{9,10}$

Pristine CNTs are known to be chemically inert-a property closely related to their high stability. As a consequence, only radicals bind strong enough to the CNT to notably affect its electrical properties. ${ }^{2,5,11-13}$ To make CNTs attractive for sensor applications thus requires some kind of functionalization, e.g., through doping or decoration of the CNT sidewall. ${ }^{13-21}$ Ideally, this type of functionalization could be used to control not only the reactivity of the CNT but also the selectivity toward specific chemical species.

In this work we consider the possibility of using CNTs doped by $3 d$ transition-metal (TM) atoms for chemical-gas sensing. We use computational screening to systematically identify the most promising dopant candidates for detection of three different target molecules $\left(\mathrm{CO}, \mathrm{NH}_{3}\right.$, and $\left.\mathrm{H}_{2} \mathrm{~S}\right)$ under typical atmospheric conditions. The screening procedure is based on the calculation of two microscopic descriptors: the binding energy and scattering resistance of the molecules when adsorbed on a doped CNT. These two quantities give a good indication of the gas coverage and impact on the resistance. For the most promising candidates we then employ a simple thermodynamic model of the CNT sensor. In this model, the binding energies are used to obtain the fractional coverage of the metallic sites as a function of the target mol- ecule concentration under ambient conditions. Under the assumption of transport in the diffusive rather than localization regime, the change in CNT resistivity may then be obtained from the calculated coverages and single impurity conductances.

Over the last few years a large number of theoretical studies on CNTs and graphene doped with transition metals have been published. ${ }^{14-21}$ Recent $\mathrm{x}$-ray absorption near edge structure experiments have demonstrated that $\mathrm{Ni}$ atoms can be present in defects of CNTs and graphene although it was not possible to identify the detailed atomic structure of the defects. ${ }^{22}$ It was also found that the $\mathrm{Ni}$ atoms were very difficult to remove from the CNT indicating a high stability of the Ni-doped defects. These experimental findings show that doping of CNTs by TM atoms is indeed possible although the doping process is difficult to control. By contrast, it has been shown that CNT vacancies, which are needed for the metallic doping, may be formed in a controlled way by irradiation by Ar ions. ${ }^{23}$

The paper is organized as follows. In Sec. II the theoretical model used in this work is discussed in detail. The computational methods are briefly described in Sec. III while the main results are discussed in Sec. IV Some final remarks are given in Sec. V In Appendix we provide a detailed comparison of the vacancy formation energies and computed structures with previous works.

\section{THEORETICAL MODEL}

In order to find the best TM-doped CNT candidates to detect the target molecules we have followed a three-steps strategy: (i) first, we have obtained the structures of the active sites, namely, the arrangement of the TM atoms in the vacancies of the CNT. (ii) Second, we have calculated the adsorption energies of the different molecules on the active sites. Based on these adsorption energies, we model the cov- 
a) Monovacancy

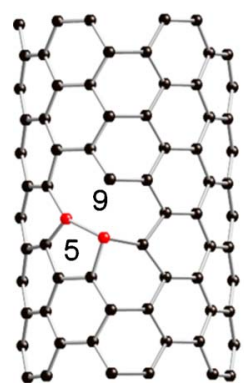

b) Divacancy 1

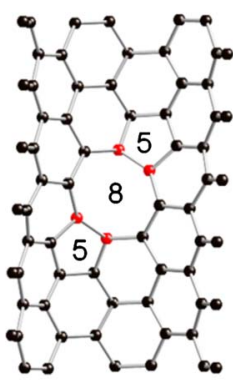

c) Divacancy II

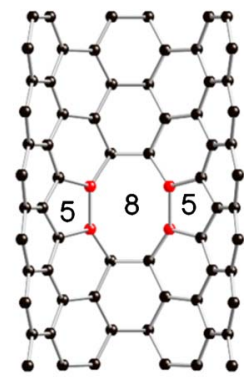

FIG. 1. (Color online) Calculated geometry reconstructions for the (a) monovacancy, (b) the divacancy I, and (c) the divacancy II in a $(6,6)$ carbon nanotube. Atoms on the far side of the nanotube are not shown for clarity. The atoms involved in the reconstructed bonds are shown in red.

erage of the active sites at thermodynamic equilibrium as a function of target molecule's concentration in air. (iii) Finally, we have calculated the changes in the electrical resistance of the CNT when a molecule is adsorbed at the active site. Combining these changes in resistance with the model coverage, we show how the resistance per active site changes with the target molecule concentration under standard atmospheric conditions. These three steps are explained in detail in this section.

\section{A. Structures}

As mentioned in the introduction, pristine CNTs are very inert and thus the adsorption of TMs on their surface would be too weak to create a stable device. In contrast the adsorption of TMs in the defects of a CNT should lead to stronger bonds that would ensure the stability of the system. In this work we have considered the adsorption of TMs in three kinds of $(6,6)$ armchair CNT defects, namely, monovacancies and the two types of divacancies (see Fig. 1). The formation energy for creating a vacancy (VC) occupied by a transitionmetal atom $(\mathrm{M})$ was calculated using the relation

$$
E_{\text {form }}[\mathrm{M} @ \mathrm{VC}]=E[\mathrm{M} @ \mathrm{VC}]-(E[\mathrm{VC}]+\mu[\mathrm{M}]),
$$

where $E[\mathrm{VC}]$ is the total energy of the nanotube with a vacancy, $E[\mathrm{M} @ \mathrm{MV}]$ is the total energy of a $\mathrm{M}$ occupying a vacancy in the CNT, and $\mu[\mathrm{M}]$ is the chemical potential of the metal atom. For the latter we have used the experimental bulk cohesive energy of the metal (enthalpy of atomization), ${ }^{24}$ which is very close to the calculated one with density-functional theory (DFT) generalized gradient approximation exchange and correlation (xc) functionals. ${ }^{25}$ The energy required to form an empty vacancy is obtained from

$$
E_{\text {form }}[\mathrm{VC}]=E[\mathrm{VC}]-n E[\mathrm{C}]-E[\mathrm{NT}],
$$

where $n$ is the number of carbon atoms removed to form the vacancy and $E[\mathrm{C}]$ is the energy per carbon atom in a pristine nanotube. The adsorption energy of a molecule $X$ is defined by

$$
\begin{aligned}
E_{\mathrm{ads}}[X @ \mathrm{M} @ \mathrm{VC}]= & E[X @ \mathrm{M} @ \mathrm{VC}] \\
& -E[\mathrm{M} @ \mathrm{VC}]-E[X],
\end{aligned}
$$

where $E[X @ \mathrm{M} @ \mathrm{VC}]$ is the total energy of molecule $X$ on a transition metal atom occupying a vacancy and $E[X]$ is the gas phase energy of the molecule.

\section{B. Coverages}

In thermodynamic equilibrium, ${ }^{26}$ the coverage of the active sites follows from

$$
\Theta[X]=\frac{K[X] C[X]}{1+\sum_{Y} K[Y] C[Y]},
$$

where $K=k_{+} / k_{-}$is the ratio of forward and backward rate constants for the adsorption reaction

$$
K[X]=\exp \left[-\frac{E_{\mathrm{ads}}+T S[X]}{k_{B} T}\right] .
$$

In these expressions $C[X]$ is the concentration of species $X$, $S[X]$ is its gas phase entropy, and $T$ is the temperature. Experimental values for the gas phase entropies have been taken from Ref. 27.

\section{Resistances}

To estimate the effect of adsorbates on the electrical conductance of doped CNTs, we first consider the change in conductance when a single molecule is adsorbed on a metal site of an otherwise pristine CNT. For the spin-polarized cases, we use the spin-averaged conductances, i.e., $G=\left(G_{\uparrow}+G_{\downarrow}\right) / 2$. Next, we estimate the resistance of a CNT containing several impurities (a specific metal dopant with different molecular adsorbates). Under the assumption that the electron phase coherence length, $l_{\phi}$, is smaller than the average distance between the dopants, $d$, we may neglect quantum interference and obtain the total resistance by adding the scattering resistances due to each impurity separately. The scattering resistance due to a single impurity is given by

$$
R_{S}[X]=\frac{1}{G[X]}-\frac{1}{2 G_{0}},
$$

where $G[X]$ is the Landauer conductance of the pristine CNT with a single metal dopant occupied by molecule $X, \frac{1}{2 G_{0}}$ is the contact resistance of a $(6,6) \mathrm{CNT}$, and $G_{0}=2 e^{2} / h$ is the quantum of conductance.

We may now obtain the total resistance per dopant site relative to the reference background signal as a function of the target molecule concentration

$$
\frac{\Delta R}{N} \approx \sum_{X} R_{S}[X]\left(\Theta[X, C]-\Theta\left[X, C_{0}\right]\right),
$$

where $N$ is the number of dopants, $\Theta[X, C]$ is the fractional coverage of species $X$ at concentration $C$ of the target, and $C_{0}$ is the reference concentration. Notice that the contact resistance drops out as we evaluate a change in resistance. 
As mentioned, the series coupling of individual scatterers should be valid when $l_{\phi}<d$. However, even for $l_{\phi}>d$ and assuming that the Anderson localization length, $l_{l o c}$ in the system exceeds $l_{\phi}$, Eq. (7) remains valid if one replaces the actual resistance $R$ by the sample averaged resistance, $\langle R\rangle{ }^{28}$ At room temperature under ambient conditions, interactions with external degrees of freedom such as internal CNT phonons and vibrational modes of the adsorbed molecules would rapidly randomize the phase of the electrons. Therefore Eq. (7) should certainly be valid in the limit of low doping concentrations. On the other hand, the total number of dopants, $N$, should be large enough for the statistical treatment of the coverage to hold. Finally, we stress that Eq. (7) represents a conservative estimate of the change in resistance. In fact, in the regime where $l_{\phi}>l_{l o c}$, i.e., in the Anderson localization regime, the resistance would be highly sensitive to changes in the fractional coverage of active sites. Calculation of the actual resistance of the CNT in this regime would, however, involve a full transport calculation in the presence of all $N$ impurities. At this point it suffices to see that the conservative estimates obtained from Eq. (7) predict measurable signals in response to small changes in concentration of the target molecules.

\section{COMPUTATIONAL DETAILS}

Metallic doping of a $(6,6)$ CNT has been modeled in a supercell containing six repeated minimal unit cells along the CNT axis (dimensions: $15 \AA \times 15 \AA \times 14.622 \AA$. Number of carbon atoms: 144.). Thus the impurity concentration has been always smaller than $1 \%$, approaching the single impurity limit. For this size of supercell a $\Gamma$-point sampling of the Brillouin zone was found to be sufficient. All total energy calculations and structure optimizations have been performed with the real-space DFT code GPAW (Refs. 29 and 30) which is based on the projector augmented wave method. Spin polarization has been taken into account in all calculations. We have only used a smearing of the electronic levels for those systems in which we found convergence problems (in any case the smearing was always lower than $0.1 \mathrm{eV}$ ). We use a grid spacing of $0.2 \AA$ for representing the density and wave functions and the Perdew-Burke-Ernzerhof (PBE) xc functional. ${ }^{31}$ Transport calculations for the optimized structures have been performed using the nonequilibrium Green's function method ${ }^{32}$ with an electronic Hamiltonian obtained from the SIESTA code ${ }^{33}$ in a double zeta polarized basis set. In all SIESTA calculations no smearing of the electronic levels was used.

\section{RESULTS AND DISCUSSION}

\section{A. Formation of vacancies and occupation by transition metals}

The first step in order to get the different adsorption energies of the TM ions in the vacancies of CNTs consists of calculating the formation energies of the vacancies. When carbon atoms are removed from a CNT an important reconstruction takes place at that site. In our calculations these reconstructions have been taken into account, by allowing

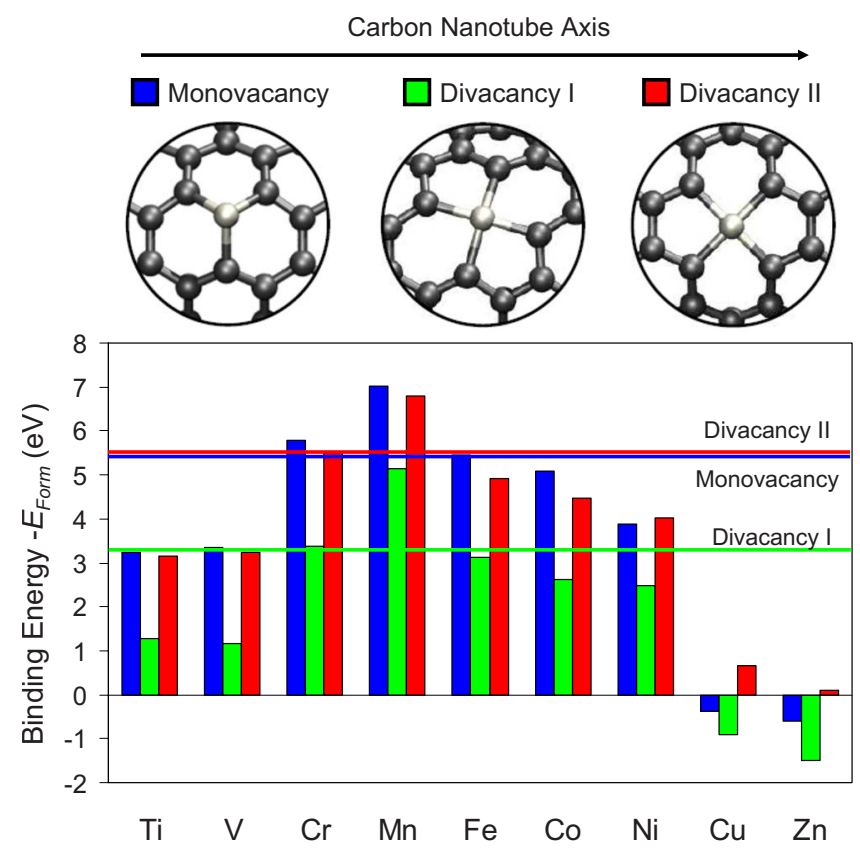

FIG. 2. (Color online) Structural schematics and binding energies (namely, formation energies with the opposite sign) for a $3 d$ transition metal occupied monovacancy (blue), divacancy I (green), or divacancy II (red) in a $(6,6)$ carbon nanotube. Binding energies of a carbon atom (two in the case of divacancies) in the empty vacancies are indicated by lines with the same color code.

the relaxation of all the carbon atoms in the unit cell. Moreover, we have checked that the relaxation of the unit-cell parameters does not influence either the reconstruction geometry or the adsorption energies. Therefore, in all the results reported in the present work, the dimensions of the unit cell have been kept fixed.

In the case of monovacancies a nonagon-pentagon structure tilted with respect to the main axis of the CNT was found to be the most stable one [see Fig. 1(a)], in good agreement with previous DFT calculations by Berber and Oshiyama. ${ }^{34}$ One of the atoms in this site becomes twofold coordinated while the other ones remain threefold coordinated.

In the case of divacancies (both divacancies I and II), a structure composed of two pentagons and one octagon is preferred with all the atoms threefold coordinated. The reconstructed bonds [see Figs. 1(b) and 1(c)] are longer in the divacancy II (DV II) than in the divacancy I (DV I) by $0.16 \AA$. This leads to a greater stability in DV I than in DV II by $2.2 \mathrm{eV}$ (see the horizontal lines in Fig. 2 and Table I in Appendix). These results are again in agreement with previous studies, ${ }^{35,36}$ as discussed in Appendix.

In Fig. 2 we show the calculated binding energies [namely, the formation energies from Eq. (1) with the opposite sign], for the different TMs in the vacancies. Thus a positive value indicates that the insertion of $\mathrm{M}$ from the metal bulk into an existing vacancy of the CNT is an exothermic process. When a transition-metal atom occupies a vacancy, the strongest bonding to the $\mathrm{C}$ atoms is through its $d$ orbitals. For this reason, $\mathrm{Cu}$ and $\mathrm{Zn}$, which both have filled $d$ bands, are rather unstable in the CNT. For the remaining 


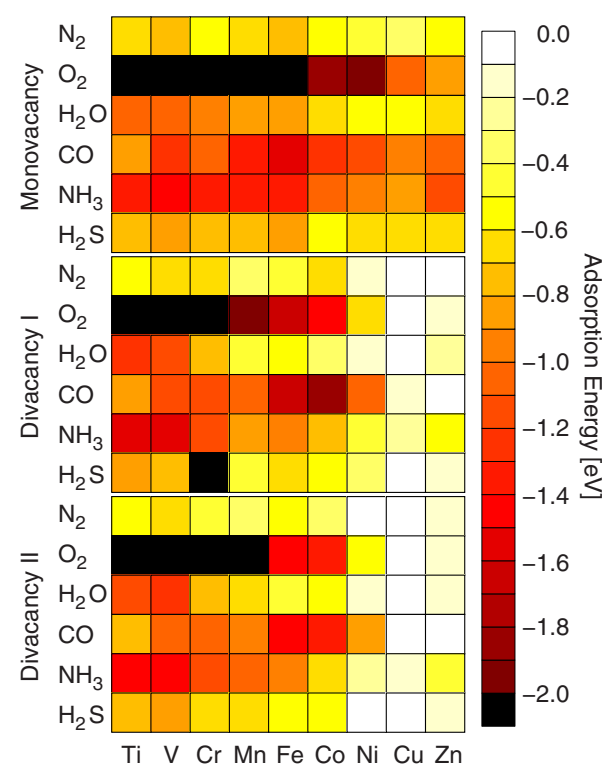

FIG. 3. (Color online) Adsorption energy, $E_{\text {ads }}$, in $\mathrm{eV}$ for $\mathrm{N}_{2}, \mathrm{O}_{2}$, $\mathrm{H}_{2} \mathrm{O}, \mathrm{CO}, \mathrm{NH}_{3}$, and $\mathrm{H}_{2} \mathrm{~S}$ on $3 d$ transition metals occupying a Monovacancy (top), Divacancy I (middle), and Divacancy II (bottom) in a $(6,6)$ carbon nanotube.

metals, adsorption in the vacancies is an exothermic process, leading to quite stable structures. Moreover, for $\mathrm{Cr}, \mathrm{Mn}, \mathrm{Fe}$, and Co the whole process of creating a vacancy and occupying it by a metal atom taken from bulk can be exothermic. This is the case when the bars in Fig. 2 are above the horizontal lines for the binding energies of $\mathrm{C}$ in the vacancies. Overall, our results are in good agreement with previous calculations by Krasheninnikov et al. $^{21}$ for metal atoms in graphene, as discussed in Appendix.

\section{B. Molecules adsorbed on top of transition metals in vacancies}

We found that the six molecules considered in the present work are always adsorbed on top of the TM atom. The adsorption does not produce significant changes on the structures described in Appendix, except for the adsorption of the $\mathrm{H}_{2} \mathrm{~S}$ molecule in the divacancy I with $\mathrm{Cr}$, in which the $\mathrm{H}_{2} \mathrm{~S}$ molecule is dissociated. However, in some cases, the electronic structure of the TM changes significantly, even producing changes in the total magnetic moment of the system (as shown in Appendix). This fact leads, as we will show in the next section, to changes in the conductance of the systems.

From the adsorption energies plotted in Fig. 3, we may estimate the fractional coverages for each available adsorbate for a given type of doping in a given background. We find that the earlier transition metals tend to bind the adsorbates stronger than the late transition metals. The latest metals in the series $(\mathrm{Cu}$ and $\mathrm{Zn})$ bind adsorbates rather weakly in the divacancy structures. We also note that $\mathrm{O}_{2}$ binds significantly stronger than any of the three target molecules on $\mathrm{Ti}, \mathrm{V}, \mathrm{Cr}$, and $\mathrm{Mn}$ (except for $\mathrm{Cr}$ in divacancy I where $\mathrm{H}_{2} \mathrm{~S}$ is found to dissociate). Active sites containing these metals are therefore expected to be completely passivated if oxygen is present in the background. Further, we find $\mathrm{H}_{2} \mathrm{O}$ is rather weakly bound

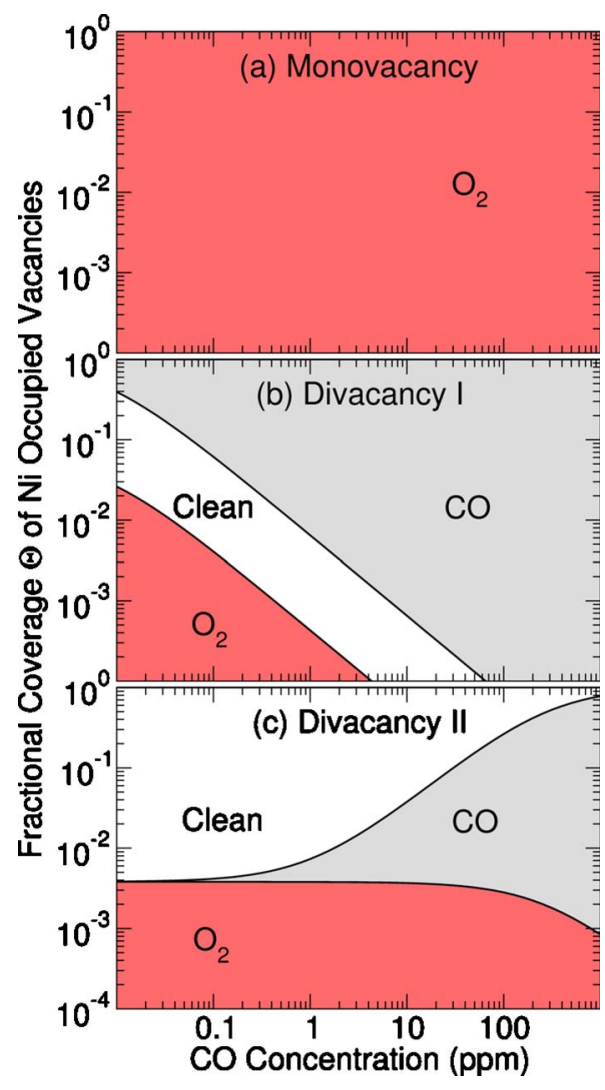

FIG. 4. (Color online) Fractional coverage, $\Theta$ in thermal equilibrium of $\mathrm{Ni}$ in a (a) monovacancy, (b) divacancy I, and (c) divacancy II, per dopant site as a function of $\mathrm{CO}$ concentration in a background of air at room temperature and 1 bar of pressure. $C_{0}$ $=0.1 \mathrm{ppm}$.

to most of the active sites. This ensures that these types of sensors are robust against changes in humidity.

From Fig. $3 \mathrm{CO}$ detection on Ni-doped CNTs seems to be the most promising candidate. Other potential systems for $\mathrm{CO}$ detection are the $\mathrm{Fe}$ - and $\mathrm{Co}$-doped vacancies. $\mathrm{Cu}$-doped CNTs could be used for $\mathrm{NH}_{3}$ detection although it is rather unstable on $\mathrm{CNT}$ vacancies. $\mathrm{H}_{2} \mathrm{~S}$ is probably not detectable with any of the dopants since it binds very weakly on all metals. Figure 4 shows the fractional coverage of a $\mathrm{Ni}$ atom occupying a monovacancy, divacancy I, and divacancy II, versus $\mathrm{CO}$ concentration in a background of air at room temperature and 1 bar of pressure. Due to the relatively small binding energy of $\mathrm{N}_{2}$ and $\mathrm{H}_{2} \mathrm{O}$ as compared to $\mathrm{O}_{2}$ and $\mathrm{CO}$, all Ni sites will be either empty or occupied by $\mathrm{O}_{2}$ or $\mathrm{CO}$. In particular, Ni in a monovacancy [see Fig. 4(a)] will be completely oxidized for all relevant $\mathrm{CO}$ concentrations. For the $\mathrm{Ni}$-occupied divacancy II structures we find the coverage of $\mathrm{CO}$ changes significantly around toxic concentrations $(\sim 50 \mathrm{ppm})$.

\section{Changes in the resistance after target molecules adsorption}

In Fig. 5 we show the calculated change in conductance relative to the metal site with no adsorbate. In contrast to the binding energies, there are no clear trends in the conductances. The sensitivity of the conductance is perhaps most 


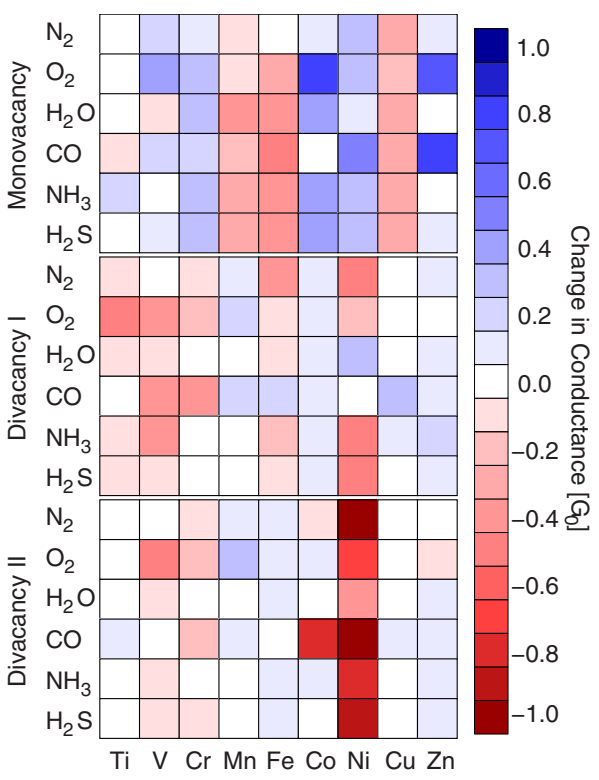

FIG. 5. (Color online) Calculated change in conductance $\Delta G$, in units of $G_{0}=2 e^{2} / h$, for $\mathrm{N}_{2}, \mathrm{O}_{2}, \mathrm{H}_{2} \mathrm{O}, \mathrm{CO}, \mathrm{NH}_{3}$, and $\mathrm{H}_{2} \mathrm{~S}$ on $3 d$ transition metals occupying a monovacancy (top), divacancy I (middle), and divacancy II (bottom) in a $(6,6)$ carbon nanotube.

clearly demonstrated by the absence of correlation between different types of vacancies, i.e., between the three panels in Fig. 5. Close to the Fermi level, the conductance of a perfect armchair CNT equals $2 G_{0}$. The presence of the metal dopant leads to several dips in the transmission function known as Fano antiresonances. ${ }^{20}$ The position and shape of these dips depend on the $d$ levels of the transition-metal atom, the character of its bonding to the CNT, and is further affected by the presence of the adsorbate molecule. The coupling of all these factors is very complex and makes it difficult to estimate or rationalize the value of the conductance.

In Sec. IV B we argued that $\mathrm{Ni}$ is the most promising dopant candidate for the detection of $\mathrm{CO}$ and, as mentioned in the introduction, $\mathrm{Ni}$ atoms have been already observed as substitutional impurities in CNTs. In Fig. 6 we show the change in resistance calculated from Eq. (7) as a function of $\mathrm{CO}$ concentration for $\mathrm{Ni}$ occupying the three types of vacancies. The background reference concentration of $\mathrm{CO}$ is taken to be $C_{0}=0.1 \mathrm{ppm}$. For the monovacancy there is very little change in resistivity. This is because most active sites are blocked by $\mathrm{O}_{2}$ at relevant $\mathrm{CO}$ concentrations, as can be seen from Fig. 4(a). For Ni in the divacancies there is, however, a change in resistance on the order of $1 \Omega$ per site. For concentrations above $\sim 1 \mathrm{ppm}$, the $\mathrm{CO}$ coverage of $\mathrm{Ni}$ in the divacancy II increases dramatically and this leads to a significant increase in resistance.

\section{CONCLUSIONS}

In summary, we have presented a general model of nanostructured chemical sensors which takes the adsorption energies of the relevant chemical species and their individual scattering resistances as the only input. For metal-doped CNTs we find that oxidation of the active metal site passi-

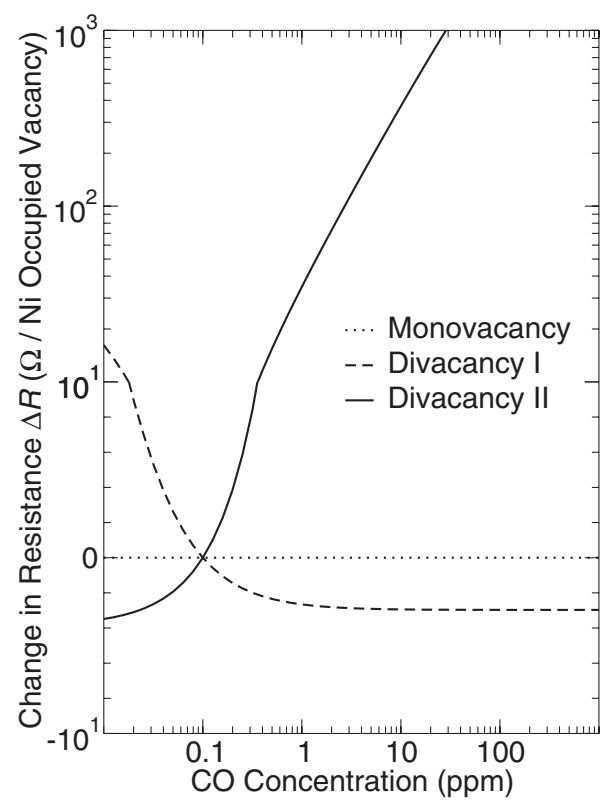

FIG. 6. Change in resistance, $\Delta R$, per Ni-occupied dopant site as a function of $\mathrm{CO}$ concentration in a background of air at room temperature and 1 bar of pressure. $C_{0}=0.1 \mathrm{ppm}$. Note the change from linear to $\log$ scale on the $y$ axis at $\Delta R=10 \Omega$.

vates the sensor in the case of doping by $\mathrm{Ti}, \mathrm{V}, \mathrm{Cr}$, and $\mathrm{Mn}$ under standard conditions (room temperature and 1 bar of pressure). Among the remaining metals, we identify $\mathrm{Ni}$, which has been observed experimentally as a substitutional atomic impurity in CNTs and graphene, ${ }^{22}$ as the most promising candidate for $\mathrm{CO}$ detection. For this system the change in resistance per active site is generally significant $(>1 \Omega)$ for small changes in $\mathrm{CO}$ concentration in the relevant range of around 0.1-10 ppm. Our approach is quite general and is directly applicable to other nanostructures than CNTs, other functionalizations than metal doping, and other backgrounds than atmospheric air.

\section{ACKNOWLEDGMENTS}

The authors acknowledge financial support from Spanish MEC (Grant No. FIS2007-65702-C02-01), ACI-Promociona (Grant No. ACI2009-1036), "Grupos Consolidados UPV/ EHU del Gobierno Vasco" (Grant No. IT-319-07), the European Union through the FP7 e-I3 ETSF (Contract No. 211956), and THEMA (Contract No. 228539) projects. They also acknowledge support by the Barcelona Supercomputing Center, "Red Española de Supercomputación," ARINA, NABIIT, and the Danish Center for Scientific Computing. The Center for Atomic-scale Materials Design (CAMD) is sponsored by the Lundbeck Foundation.

\section{APPENDIX}

\section{Vacancy formation energies}

In Table I our results are compared with the ones from Berber and Oshiyama. ${ }^{34,35}$ Berber and Oshiyama used the 
TABLE I. Calculated formation energies and reconstructed bond lengths of the different vacancies in a $(6,6)$ carbon nanotube. The results from Berber and Oshiyama can be found in Ref. 34 for the monovacancy and in the Ref. 35 for both divacancies.

\begin{tabular}{lccccc}
\hline \hline & \multicolumn{2}{c}{$\begin{array}{c}\text { Formation } \\
\text { Energies } \\
(\mathrm{eV})\end{array}$} & & \multicolumn{2}{c}{$\begin{array}{c}\text { Reconstructed } \\
\text { bond lengths } \\
(\AA)\end{array}$} \\
\cline { 2 - 3 } \cline { 5 - 6 } & This work & Berber et al. & This work & Berber et al. \\
\hline Monovacancy & 5.4 & $\sim 5.5$ & & 1.55 & 1.48 \\
Divacancy I & 3.3 & $\sim 4$ & & 1.49 & 1.60 \\
Divacancy II & 5.5 & $\sim 7$ & & 1.65 & 1.60 \\
\hline \hline
\end{tabular}

same expression for the formation energies as we did. They used the local-density approximation (LDA) approximation ${ }^{37}$ and a double- $\zeta$ polarized basis set within the SIESTA code. ${ }^{33}$ The trends in the calculated formation energies are in good agreement. The discrepancies are due to two factors: (i) our calculations are based on a real-space code while Berber and Oshiyama used a local basis set approximation. (ii) We use the PBE xc functional ${ }^{31}$ rather than the LDA which is known to overestimate formation energies. ${ }^{38}$

\section{Structure of transition-metal-doped vacancies}

When a TM atom is adsorbed in a monovacancy of a $(6,6)$ CNT it may be considered as a substitutional impurity, in the sense that the TM atom replaces a $\mathrm{C}$ atom and does not produce drastic changes in the CNT structure. All the metal atoms considered in this work form covalent bonds with three carbons (the same three carbons that were bonded to the substituted carbon). Upon adsorption the pentagon structure formed in the reconstruction of the monovacancy disappears. As the TM atomic radii are larger than that of the carbon atom, the metal atoms displace outward from the CNT surface. The TM-C bond length decreases from Ti to Fe as the atom size decreases (see Table II) then it increases as the bonding becomes weaker for the more noble metals.

Our structures and bond distances (Table II) are very close to those reported by Krasheninnikov et al. ${ }^{21}$ for the same TMs in graphene. Krasheninnikov et al. performed DFT calculations, using the PBE exchange-correlation functional ${ }^{31}$ and a plane-wave code.

In graphene, the three $\mathrm{C}$ atoms next to the $\mathrm{TM}$ are equivalent. However, due to curvature effects in the $(6,6) \mathrm{CNT}$ two of the TM-C bonds are shorter than the remaining one, see Figs. $7(\mathrm{~b})$ and $7(\mathrm{~g})$. For most of the TM atoms the difference between the short and long bonds is in the range 0.04-0.06 ̊.

For the case of the divacancies in a $(6,6) \mathrm{CNT}$ the TM atoms form a "cross configuration" and are bonded to four carbon atoms. As it happened for the monovacancies, the pentagon-octagon-pentagon reconstruction disappears, see Figs. 7(c)-7(f). The obtained structures and bond distances are again very similar to those found by Krasheninnikov et $a l .{ }^{21}$ in graphene, except for the $\mathrm{V}$ atom. Krasheninnikov et $a l .{ }^{21}$ found that $\mathrm{V}$ prefers to bind to only two carbon atoms in a)

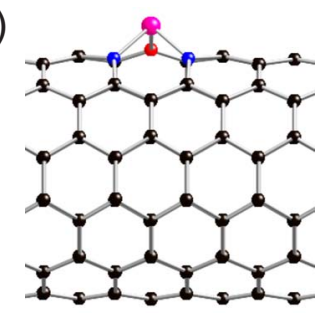

c)

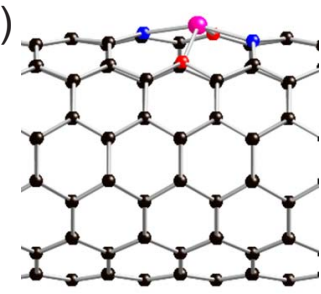

e)

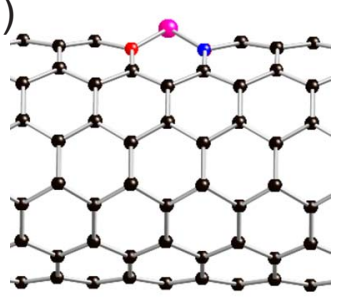

b)

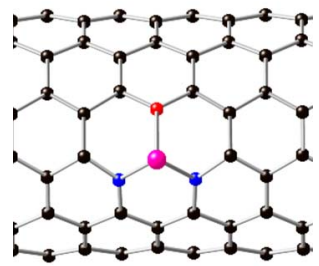

d)

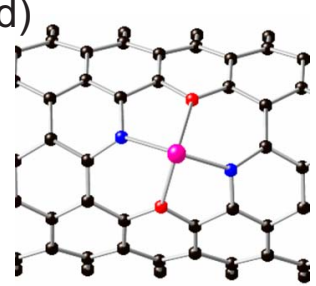

f)

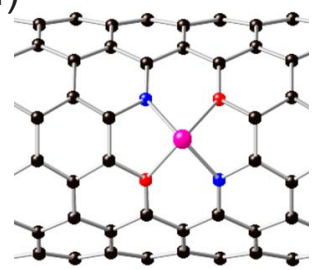

g) Long-short bond difference

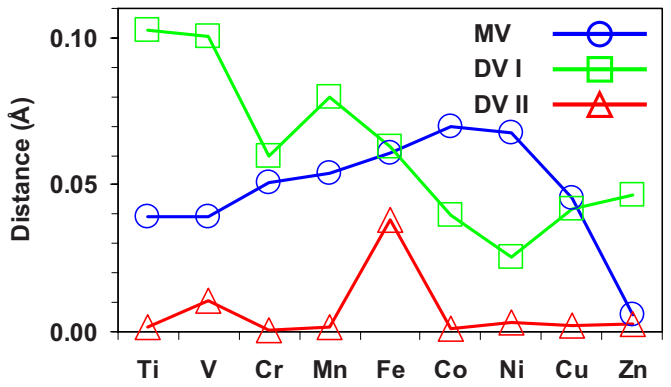

FIG. 7. (Color online) $[(\mathrm{a})-(\mathrm{f})]$ Structures of a $(6,6) \mathrm{CNT}$ with a transition-metal atom (pink atom) adsorbed in a monovacancy and in the two divacancies considered in this work. Monovacancy is showed in (a) (side view) and (b) (top view). Divacancy I is showed in (c) (side view) and (d) (top view). Divacancy II is showed in (e) (side view) and (f) (top view). In all the figures the carbon atoms bonded to the TM are highlighted in red (longer bonds) and blue (shorter bonds). (g) Difference in bond length between the two inequivalent TM-C bonds formed around the TM-doped monovacancies (blue circles), divacancies I (green squares), and divacancies II (red triangles).

a graphene divacancy. In contrast, we found that the $\mathrm{V}$ atom also forms a cross configuration in the divacancies of a $(6,6)$ CNT, as do the rest of the TM atoms considered in this work. Since there is more available space in the divacancies than in the monovacancy, the TM-C distance is larger in the former than in the latter. For the same reason the TM atoms are closer to the CNT surface in the divacancies than in the monovacancies. The TM-C bond length follows a similar trend along the Ti-Zn series in divacancies as found for the monovacancies. There are only two differences: (i) the shorter TM-C distance in the monovacancy series is for $\mathrm{Fe}$ whereas it is for $\mathrm{Ni}$ in the divacancies. (ii) $\mathrm{Fe}, \mathrm{Co}$, and $\mathrm{Ni}$ are 
TABLE II. Comparison between the calculated average transition metal-carbon bond lengths in the different vacancies in a $(6,6)$ carbon nanotube (D) and the total magnetic moment of the unit cells (M) from this work and the calculated ones by Krasheninnikov et al. (Ref. 21) in graphene. Notice that in graphene there is only one type of divacancy. Distances and magnetic moments are given in $\AA$ and $\mu_{\mathrm{B}}$, respectively.

\begin{tabular}{|c|c|c|c|c|c|c|c|c|c|c|}
\hline & \multicolumn{4}{|c|}{ Monovacancy } & \multicolumn{6}{|c|}{ Divacancy } \\
\hline & \multicolumn{2}{|c|}{ This work } & \multicolumn{2}{|c|}{ Krasheninnikov et al. (Ref. 21) } & \multicolumn{2}{|c|}{ This work DV I } & \multicolumn{2}{|c|}{ This work DV II } & \multicolumn{2}{|c|}{ Krasheninnikov et al. (Ref. 21) } \\
\hline & $\mathrm{D}$ & M & $\mathrm{D}$ & $\mathrm{M}$ & $\mathrm{D}$ & M & $\mathrm{D}$ & $\mathrm{M}$ & $\mathrm{D}$ & M \\
\hline $\mathrm{Ti}$ & 1.95 & 0.0 & 1.95 & 0.0 & 2.10 & 0.0 & 2.05 & 0.0 & 2.07 & 0.0 \\
\hline $\mathrm{V}$ & 1.92 & 1.0 & 1.88 & 1.0 & 2.07 & 1.0 & 2.00 & 1.0 & 2.14 & 3.0 \\
\hline $\mathrm{Cr}$ & 1.89 & 2.0 & 1.86 & 2.0 & 2.02 & 2.0 & 1.97 & 2.0 & 2.02 & 2.0 \\
\hline $\mathrm{Mn}$ & 1.85 & 3.0 & 1.83 & 2.7 & 2.01 & 3.0 & 1.96 & 3.0 & 1.99 & 3.0 \\
\hline $\mathrm{Fe}$ & 1.79 & 2.0 & 1.76 & 0.0 & 1.96 & 4.0 & 1.90 & 4.0 & 1.96 & 3.3 \\
\hline Co & 1.79 & 1.0 & 1.77 & 1.0 & 1.92 & 1.0 & 1.89 & 1.0 & 1.94 & 1.3 \\
\hline $\mathrm{Ni}$ & 1.82 & 0.0 & 1.78 & 0.0 & 1.89 & 0.0 & 1.85 & 0.0 & 1.89 & 0.0 \\
\hline $\mathrm{Cu}$ & 1.86 & 1.0 & 1.87 & 1.4 & 1.93 & 1.0 & 1.87 & 1.0 & 1.91 & 0.0 \\
\hline $\mathrm{Zn}$ & 1.92 & 0.0 & 1.88 & 0.0 & 1.97 & 0.0 & 1.90 & 0.0 & 1.94 & 0.0 \\
\hline
\end{tabular}

TABLE III. Calculated distances between the transition-metal atom and the closest $\mathrm{N}$ atom of the $\mathrm{N}_{2}$ molecule, TM- $\mathrm{N}_{2}$, and calculated average distances between the transition-metal atom and the closest $\mathrm{C}$ atoms of the CNT (see Fig. 7), TM-C. The total magnetic moment of the system is also shown. Distances and magnetic moments are given in $\AA$ and $\mu_{\mathrm{B}}$ units, respectively.

\begin{tabular}{|c|c|c|c|c|c|c|c|c|c|}
\hline & \multicolumn{3}{|c|}{ Monovacancy } & \multicolumn{3}{|c|}{ Divacancy I } & \multicolumn{3}{|c|}{ Divacancy II } \\
\hline & TM- $\mathrm{N}_{2}$ & TM-C & Spin & TM-N ${ }_{2}$ & TM-C & Spin & TM- $\mathrm{N}_{2}$ & TM-C & Spin \\
\hline $\mathrm{Ti}$ & 2.20 & 1.97 & 2.0 & 2.21 & 2.09 & 0.0 & 2.15 & 1.61 & 0.0 \\
\hline V & 2.10 & 1.93 & 1.0 & 2.07 & 1.77 & 1.0 & 2.08 & 1.73 & 1.0 \\
\hline $\mathrm{Cr}$ & 2.07 & 1.90 & 2.0 & 1.99 & 1.99 & 2.0 & 2.02 & 2.00 & 2.0 \\
\hline $\mathrm{Mn}$ & 1.98 & 1.85 & 3.0 & 2.01 & 2.21 & 3.0 & 2.00 & 2.30 & 3.0 \\
\hline $\mathrm{Fe}$ & 1.90 & 1.82 & 2.3 & 1.90 & 1.99 & 2.0 & 1.86 & 1.94 & 2.0 \\
\hline $\mathrm{Co}$ & 1.89 & 1.82 & 1.0 & 1.80 & 1.63 & 1.0 & 1.87 & 1.58 & 1.1 \\
\hline $\mathrm{Ni}$ & 1.89 & 1.85 & 0.0 & 1.90 & 1.41 & 0.0 & 1.94 & 1.31 & 2.0 \\
\hline $\mathrm{Cu}$ & 1.95 & 1.92 & 1.0 & 3.07 & 1.98 & 1.0 & 2.81 & 2.22 & 1.0 \\
\hline $\mathrm{Zn}$ & 2.00 & 1.95 & 0.0 & 3.05 & 1.75 & 0.0 & 2.77 & 1.89 & 0.0 \\
\hline
\end{tabular}

TABLE IV. Calculated distances between the transition-metal atom and the closest $\mathrm{O}$ atom of the $\mathrm{O}_{2}$ molecule, TM- $\mathrm{O}_{2}$, and calculated average distances between the transition-metal atom and the closest $\mathrm{C}$ atoms of the CNT (see Fig. 7), TM-C. The total magnetic moment of the system is also shown. Distances and magnetic moments are given in $\AA$ and $\mu_{\mathrm{B}}$ units, respectively.

\begin{tabular}{|c|c|c|c|c|c|c|c|c|c|}
\hline & \multicolumn{3}{|c|}{ Monovacancy } & \multicolumn{3}{|c|}{ Divacancy I } & \multicolumn{3}{|c|}{ Divacancy II } \\
\hline & TM-O ${ }_{2}$ & TM-C & Spin & TM-O ${ }_{2}$ & TM-C & Spin & $\mathrm{TM}-\mathrm{O}_{2}$ & TM-C & Spin \\
\hline $\mathrm{Ti}$ & 1.87 & 2.08 & 0.0 & 1.93 & 2.16 & 0.0 & 1.97 & 2.12 & 2.0 \\
\hline V & 1.88 & 1.97 & 1.0 & 1.87 & 2.09 & 1.0 & 1.89 & 2.05 & 1.0 \\
\hline $\mathrm{Cr}$ & 1.84 & 1.91 & 1.1 & 1.84 & 2.03 & 0.8 & 1.87 & 1.99 & 0.0 \\
\hline $\mathrm{Mn}$ & 1.84 & 1.89 & 1.9 & 1.84 & 2.00 & 1.1 & 1.87 & 1.97 & 2.3 \\
\hline $\mathrm{Fe}$ & 1.86 & 1.85 & 2.0 & 1.87 & 2.01 & 2.0 & 1.74 & 1.93 & 2.0 \\
\hline $\mathrm{Co}$ & 1.88 & 1.83 & 1.0 & 1.89 & 1.95 & 0.4 & 1.95 & 1.92 & 0.9 \\
\hline $\mathrm{Ni}$ & 1.86 & 1.86 & 1.2 & 1.94 & 1.94 & 0.7 & 2.02 & 1.93 & 0.9 \\
\hline $\mathrm{Cu}$ & 1.82 & 1.94 & 1.0 & 2.82 & 1.93 & 1.0 & 3.45 & 1.87 & 3.0 \\
\hline $\mathrm{Zn}$ & 1.97 & 1.98 & 0.0 & 2.45 & 1.98 & 0.0 & 2.63 & 1.91 & 0.0 \\
\hline
\end{tabular}


TABLE V. Calculated distances between the transition-metal atom and the $\mathrm{O}$ atom of the $\mathrm{H}_{2} \mathrm{O}$ molecule, TM- $\mathrm{H}_{2} \mathrm{O}$, and calculated average distances between the transition-metal atom and the closest $\mathrm{C}$ atoms of the CNT (see Fig. 7), TM-C. The total magnetic moment of the system is also shown. Distances and magnetic moments are given in $\AA$ and $\mu_{\mathrm{B}}$ units, respectively.

\begin{tabular}{|c|c|c|c|c|c|c|c|c|c|}
\hline & \multicolumn{3}{|c|}{ Monovacancy } & \multicolumn{3}{|c|}{ Divacancy I } & \multicolumn{3}{|c|}{ Divacancy II } \\
\hline & $\mathrm{TM}-\mathrm{H}_{2} \mathrm{O}$ & TM-C & Spin & $\mathrm{TM}-\mathrm{H}_{2} \mathrm{O}$ & TM-C & Spin & $\mathrm{TM}-\mathrm{H}_{2} \mathrm{O}$ & TM-C & Spin \\
\hline $\mathrm{Ti}$ & 2.24 & 1.97 & 0.6 & 2.16 & 2.11 & 0.0 & 2.19 & 2.06 & 0.0 \\
\hline V & 2.18 & 1.93 & 1.0 & 2.13 & 2.07 & 1.0 & 2.13 & 2.00 & 1.0 \\
\hline $\mathrm{Cr}$ & 2.13 & 1.89 & 2.0 & 2.11 & 2.02 & 2.0 & 2.15 & 1.97 & 2.0 \\
\hline Mn & 2.09 & 1.84 & 3.0 & 2.24 & 2.02 & 3.0 & 2.19 & 1.96 & 3.0 \\
\hline $\mathrm{Fe}$ & 2.07 & 1.80 & 2.0 & 2.20 & 1.98 & 4.0 & 2.16 & 1.93 & 4.0 \\
\hline Co & 2.10 & 1.80 & 1.0 & 2.19 & 1.93 & 1.0 & 2.16 & 1.90 & 1.7 \\
\hline $\mathrm{Ni}$ & 2.16 & 1.84 & 0.0 & 2.52 & 1.90 & 0.0 & 2.70 & 1.85 & 0.0 \\
\hline $\mathrm{Cu}$ & 2.14 & 1.89 & 1.0 & 2.95 & 1.93 & 1.0 & 3.07 & 1.87 & 1.0 \\
\hline $\mathrm{Zn}$ & 2.14 & 1.96 & 0.0 & 2.42 & 1.98 & 0.0 & 2.52 & 1.92 & 0.0 \\
\hline
\end{tabular}

TABLE VI. Calculated distances between the transition-metal atom and the $\mathrm{C}$ atom of the $\mathrm{CO}$ molecule, TM-CO, and calculated average distances between the transition-metal atom and the closest $\mathrm{C}$ atoms of the CNT (see Fig. 7), TM-C. The total magnetic moment of the system is also shown. Distances and magnetic moments are given in $\AA$ and $\mu_{\mathrm{B}}$ units, respectively.

\begin{tabular}{|c|c|c|c|c|c|c|c|c|c|}
\hline & \multicolumn{3}{|c|}{ Monovacancy } & \multicolumn{3}{|c|}{ Divacancy I } & \multicolumn{3}{|c|}{ Divacancy II } \\
\hline & TM-CO & TM-C & Spin & TM-CO & TM-C & Spin & TM-CO & TM-C & Spin \\
\hline $\mathrm{Ti}$ & 2.22 & 1.98 & 2.0 & 2.25 & 2.11 & 0.0 & 2.21 & 2.05 & 0.0 \\
\hline $\mathrm{V}$ & 2.10 & 1.93 & 1.0 & 2.02 & 2.06 & 3.0 & 2.07 & 2.01 & 3.0 \\
\hline $\mathrm{Cr}$ & 2.01 & 1.88 & 2.0 & 1.94 & 2.03 & 4.0 & 1.98 & 1.97 & 2.0 \\
\hline Mn & 1.89 & 1.86 & 3.0 & 1.81 & 1.97 & 3.0 & 1.93 & 1.96 & 3.0 \\
\hline $\mathrm{Fe}$ & 1.82 & 1.83 & 2.0 & 1.73 & 1.95 & 2.0 & 1.80 & 1.92 & 0.0 \\
\hline Co & 1.80 & 1.84 & 1.0 & 1.71 & 1.92 & 1.0 & 1.70 & 1.87 & 1.0 \\
\hline $\mathrm{Ni}$ & 1.84 & 1.86 & 0.0 & 1.78 & 1.92 & 0.0 & 1.79 & 1.88 & 2.0 \\
\hline $\mathrm{Cu}$ & 1.87 & 1.94 & 1.0 & 2.02 & 1.96 & 1.0 & 2.11 & 1.90 & 1.0 \\
\hline $\mathrm{Zn}$ & 1.89 & 1.96 & 0.0 & 2.36 & 1.99 & 0.0 & 2.58 & 1.91 & 0.0 \\
\hline
\end{tabular}

TABLE VII. Calculated distances between the transition-metal atom and the $\mathrm{N}$ atom of the $\mathrm{NH}_{3}$ molecule, $\mathrm{TM}^{-\mathrm{NH}_{3}}$, and calculated average distances between the transition-metal atom and the closest $\mathrm{C}$ atoms of the CNT (see Fig. 7), TM-C. The total magnetic moment of the system is also shown. Distances and magnetic moments are given in $\AA$ and $\mu_{\mathrm{B}}$ units, respectively.

\begin{tabular}{|c|c|c|c|c|c|c|c|c|c|}
\hline & \multicolumn{3}{|c|}{ Monovacancy } & \multicolumn{3}{|c|}{ Divacancy I } & \multicolumn{3}{|c|}{ Divacancy II } \\
\hline & TM-NH 3 & TM-C & Spin & $\mathrm{TM}^{-\mathrm{NH}_{3}}$ & TM-C & Spin & TM-NH 3 & TM-C & Spin \\
\hline $\mathrm{Ti}$ & 2.31 & 1.97 & 0.0 & 2.21 & 2.10 & 0.0 & 2.23 & 2.05 & 0.0 \\
\hline V & 2.23 & 1.93 & 1.0 & 2.19 & 2.06 & 1.0 & 2.20 & 2.01 & 1.0 \\
\hline $\mathrm{Cr}$ & 2.18 & 1.89 & 2.0 & 2.13 & 2.02 & 4.0 & 2.15 & 1.97 & 2.0 \\
\hline $\mathrm{Mn}$ & 2.12 & 1.84 & 3.0 & 2.17 & 2.02 & 3.0 & 2.17 & 1.96 & 3.0 \\
\hline $\mathrm{Fe}$ & 2.09 & 1.80 & 2.0 & 2.16 & 1.98 & 4.0 & 2.14 & 1.93 & 4.0 \\
\hline $\mathrm{Co}$ & 2.09 & 1.80 & 1.0 & 2.06 & 1.92 & 1.5 & 2.14 & 1.91 & 3.0 \\
\hline $\mathrm{Ni}$ & 2.11 & 1.84 & 0.0 & 2.10 & 1.91 & 0.0 & 2.14 & 1.87 & 2.0 \\
\hline $\mathrm{Cu}$ & 2.10 & 1.91 & 1.0 & 2.31 & 1.94 & 1.0 & 2.43 & 1.88 & 1.0 \\
\hline $\mathrm{Zn}$ & 2.06 & 1.98 & 0.0 & 2.21 & 2.00 & 0.0 & 2.30 & 1.93 & 0.0 \\
\hline
\end{tabular}


TABLE VIII. Calculated distances between the transition-metal atom and the $\mathrm{S}$ atom of the $\mathrm{H}_{2} \mathrm{~S}$ molecule, TM- $\mathrm{H}_{2} \mathrm{~S}$, and calculated average distances between the transition-metal atom and the closest $\mathrm{C}$ atoms of the CNT (see Fig. 7), TM-C. The total magnetic moment of the system is also shown. Distances and magnetic moments are given in $\AA$ and $\mu_{\mathrm{B}}$ units, respectively.

\begin{tabular}{|c|c|c|c|c|c|c|c|c|c|}
\hline & \multicolumn{3}{|c|}{ Monovacancy } & \multicolumn{3}{|c|}{ Divacancy I } & \multicolumn{3}{|c|}{ Divacancy II } \\
\hline & $\mathrm{TM}-\mathrm{H}_{2} \mathrm{~S}$ & TM-C & Spin & $\mathrm{TM}-\mathrm{H}_{2} \mathrm{~S}$ & TM-C & Spin & TM- $\mathrm{H}_{2} \mathrm{~S}$ & TM-C & Spin \\
\hline $\mathrm{Ti}$ & 2.73 & 1.97 & 0.6 & 2.63 & 2.10 & 0.0 & 2.64 & 2.05 & 0.0 \\
\hline V & 2.61 & 1.93 & 1.0 & 2.48 & 2.06 & 1.0 & 2.57 & 2.00 & 1.0 \\
\hline $\mathrm{Cr}$ & 2.53 & 1.89 & 2.0 & 2.22 & 2.14 & 2.0 & 2.52 & 1.97 & 2.0 \\
\hline $\mathrm{Mn}$ & 2.48 & 1.84 & 3.0 & 2.53 & 2.02 & 3.0 & 2.51 & 1.96 & 3.0 \\
\hline $\mathrm{Fe}$ & 2.32 & 1.80 & 2.0 & 2.37 & 1.97 & 4.0 & 2.42 & 1.92 & 4.0 \\
\hline $\mathrm{Co}$ & 2.43 & 1.80 & 1.0 & 2.28 & 1.92 & 1.0 & 2.35 & 1.91 & 1.7 \\
\hline $\mathrm{Ni}$ & 2.32 & 1.84 & 0.0 & 2.33 & 1.91 & 0.0 & 2.36 & 1.87 & 0.0 \\
\hline $\mathrm{Cu}$ & 2.32 & 1.92 & 1.0 & 3.37 & 1.92 & 1.0 & 3.38 & 1.87 & 1.0 \\
\hline $\mathrm{Zn}$ & 2.40 & 1.95 & 0.0 & 3.86 & 1.98 & 0.0 & 2.95 & 1.91 & 0.0 \\
\hline
\end{tabular}

ions with a notably shorter TM-C distance in the monovacancy than the rest of the TMs. In the divacancies, $\mathrm{Cu}$ atom also belongs to this group of short TM-C distances. This is due to the well-known tendency of $\mathrm{Cu}$ and $\mathrm{Ni}$ atoms to form tetracoordinated transition-metal complexes. ${ }^{39}$

There are two main differences between the adsorption of TM atoms in divacancies of type I and type II: (i) the average TM-C distances are shorter in DV II than in DV I (see Table II). This is because, due to the CNT curvature, the geometry in the DV II is closer to a square-planar transition-metal complex structure (this is a preferred geometry for many TM atoms $^{39}$ ) than in DV I. (ii) In DV I, again due to the CNT curvature, the two TM-C bonds along the main CNT axis direction are shorter than the other two directions [see Fig. $7(\mathrm{~g})]$. The difference between the short and the long TM-C distances varies significantly along the TM series (from 3 to $10 \mathrm{pm}$ ). On the other hand, in DV II the four TM-C bonds are equivalent by symmetry and thus there are no differences in the distances between short and long bonds, except for Fe. For the Fe atom the symmetry is reduced due to the JahnTeller effect ${ }^{39}$ and there is a slight difference of $3.5 \mathrm{pm}$ between the short and the long bonds [see Fig. $7(\mathrm{~g})]$.

\section{Molecular adsorption geometries}

Several initial guess geometries have been considered for each studied molecule. Possible dissociations of $\mathrm{H}_{2} \mathrm{O}, \mathrm{H}_{2} \mathrm{~S}$, and $\mathrm{NH}_{3}$ molecules have been taken into account. In the case of $\mathrm{CO}$ the two possible bonds (through $\mathrm{C}$ atom or through $\mathrm{O}$ atom) with the TM have been analyzed.

The $\mathrm{N}_{2}$ molecule is adsorbed vertically, forming a covalent bond between the TM atom and one of the $\mathrm{N}$ atoms of the molecule (see Table III). The $\mathrm{O}_{2}$ molecule is adsorbed parallel to the plane formed by the closest $\mathrm{C}$ atoms of the CNT, forming two covalent bonds between the TM atom and each of the $\mathrm{O}$ atoms of the molecule (see Table IV). Only in the case of the $\mathrm{Zn}$ atom in the monovacancy, the $\mathrm{O}_{2}$ molecule is adsorbed vertically. The $\mathrm{H}_{2} \mathrm{O}$ molecule forms a covalent bond between the TM atom and the $\mathrm{O}$ atom of the molecule (see Table V). The CO molecule is adsorbed vertically, forming a covalent bond between the TM atom and the $\mathrm{C}$ atom of the molecule (see Table VI). The $\mathrm{NH}_{3}$ molecule forms a covalent bond between the TM atom and the $\mathrm{N}$ atom of the molecule (see Table VII). The $\mathrm{H}_{2} \mathrm{~S}$ molecule forms a covalent bond between the TM atom and the $\mathrm{S}$ atom of the molecule (see Table VIII). In the case of $\mathrm{CO}, \mathrm{NH}_{3}$ and $\mathrm{N}_{2}$, the arrangements of the molecules are similar to those found by Yeung et al. ${ }^{18}$ on $\mathrm{Pt}$ atoms placed in monovacancies for the same three molecules.

We have observed the following four trends in the adsorption mechanism: (i) the shorter distances between the TM and the molecule are for $\mathrm{Fe}, \mathrm{Co}$ and $\mathrm{Ni}$ (except for the $\mathrm{H}_{2} \mathrm{O}$ on $\mathrm{Ni}$ ). This is expected from the atomic radii of the considered TM atoms. It is worth noting to mention that the interaction of Fe with CNT is one of the most widely studied in the literature. ${ }^{14,19,40}$ (ii) The distances between the molecules and $\mathrm{Cu}$ or $\mathrm{Zn}$ are significantly larger than for the other TM atoms (in some cases by more than $1 \AA$ ) in the divacancies. This behavior is also observed for the $\mathrm{H}_{2} \mathrm{O}$ molecule on Ni. This is due to the aforementioned tendency of these TM atoms to form square planar transition-metal complexes. ${ }^{39}$ (iii) The order in the average TM-molecule distances along the TM series is the following for monovacancies: $\mathrm{H}_{2} \mathrm{~S}$ $>\mathrm{H}_{2} \mathrm{O} \sim \mathrm{NH}_{3}>\mathrm{N}_{2}>\mathrm{CO}>\mathrm{O}_{2}$ and divacancies: $\mathrm{H}_{2} \mathrm{~S}>\mathrm{H}_{2} \mathrm{O}$ $>\mathrm{NH}_{3}>\mathrm{N}_{2}>\mathrm{O}_{2}>\mathrm{CO}$ (see Tables III-VIII). The main difference between monovacancies and divacancies is that the $\mathrm{O}_{2}$ molecule forms the shortest bonds when adsorbed on TM atoms in a monovacancy while the $\mathrm{CO}$ molecule does this for the divacancies. (iv) After the adsorption of the molecules the TM-C distances increases with respect to the case of a clean TM by 2-3 pm for $\mathrm{N}_{2}, \mathrm{H}_{2} \mathrm{O}, \mathrm{CO}, \mathrm{NH}_{3}$, and $\mathrm{H}_{2} \mathrm{~S}$ (compare Table III-VIII with Table II). This is because the bond order of the TM atom increases by one after the adsorption. In the case of the $\mathrm{O}_{2}$ molecules this increase in the TM-C distance is about 5-6 pm, because it forms two bonds with the TM atoms, instead of one, as the rest of the molecules do. 
${ }^{1}$ Gas Sensing Materials, special issue, MRS Bull. Vol. 24, No. 6 (1999).

${ }^{2}$ J. C. Charlier, X. Blase, and S. Roche, Rev. Mod. Phys. 79, 677 (2007).

${ }^{3}$ J. Kong, N. R. Franklin, C. W. Zhou, M. G. Chapline, S. Peng, K. J. Cho, and H. J. Dai, Science 287, 622 (2000).

${ }^{4}$ P. G. Collins, K. Bradley, M. Ishigami, and A. Zettl, Science 287, 1801 (2000).

${ }^{5}$ C. Hierold, Carbon Nanotube Devices: Properties, Modeling, Integration and Applications (Wiley-VCH, Weinheim, 2008).

${ }^{6}$ F. Villalpando-Páez, A. H. Romero, E. Muñoz-Sandoval, L. M. Martínez, H. Terrones, and M. Terrones, Chem. Phys. Lett. 386, 137 (2004)

${ }^{7}$ A. R. Rocha, M. Rossi, A. Fazzio, and A. J. R. da Silva, Phys. Rev. Lett. 100, 176803 (2008).

${ }^{8}$ S. Brahim, S. Colbern, R. Gump, and L. Grigorian, J. Appl. Phys. 104, 024502 (2008).

${ }^{9}$ C. Morgan, Z. Alemipour, and M. Baxendale, Phys. Status Solidi A 205, 1394 (2008).

${ }^{10}$ D. J. Mowbray, C. Morgan, and K. S. Thygesen, Phys. Rev. B 79, 195431 (2009).

${ }^{11}$ L. Valentini, F. Mercuri, I. Armentano, C. Cantalini, S. Picozzi, L. Lozzi, S. Santucci, A. Sgamellotti, and J. M. Kenny, Chem. Phys. Lett. 387, 356 (2004)

${ }^{12}$ Z. Zanolli and J. C. Charlier, Phys. Rev. B 80, 155447 (2009).

${ }^{13}$ J. M. García-Lastra, K. S. Thygesen, M. Strange, and Á. Rubio, Phys. Rev. Lett. 101, 236806 (2008).

${ }^{14}$ S. B. Fagan, R. Mota, A. J. R. da Silva, and A. Fazzio, Phys. Rev. B 67, 205414 (2003).

${ }^{15}$ Y. Yagi, T. M. Briere, M. H. F. Sluiter, V. Kumar, A. A. Farajian, and Y. Kawazoe, Phys. Rev. B 69, 075414 (2004).

${ }^{16}$ S. H. Yang, W. H. Shin, J. W. Lee, S. Y. Kim, S. I. Woo, and J. K. Kang, J. Phys. Chem. B 110, 13941 (2006).

${ }^{17}$ K. T. Chan, J. B. Neaton, and M. L. Cohen, Phys. Rev. B 77, 235430 (2008).

${ }^{18}$ C. S. Yeung, L. V. Liu, and Y. A. Wang, J. Phys. Chem. C 112, 7401 (2008)

${ }^{19}$ T. Vo, Y.-D. Wu, R. Car, and M. Robert, J. Phys. Chem. C 112 , 8400 (2008)

${ }^{20}$ J. A. Fürst, M. Brandbyge, A.-P. Jauho, and K. Stokbro, Phys. Rev. B 78, 195405 (2008).
${ }^{21}$ A. V. Krasheninnikov, P. O. Lehtinen, A. S. Foster, P. Pyykkö, and R. M. Nieminen, Phys. Rev. Lett. 102, 126807 (2009).

${ }^{22}$ M. Ushiro, K. Uno, T. Fujikawa, Y. Sato, K. Tohji, F. Watari, W. J. Chun, Y. Koike, and K. Asakura, Phys. Rev. B 73, 144103 (2006).

${ }^{23}$ C. Gómez-Navarro, P. J. De Pablo, J. Gómez-Herrero, B. Biel, F. J. Garcia-Vidal, A. Rubio, and F. Flores, Nature Mater. 4, 534 (2005).

${ }^{24}$ C. Kittel, Introduction to Solid State Physics (Wiley, New York, 2004).

${ }^{25}$ P. H. T. Philipsen and E. J. Baerends, Phys. Rev. B 54, 5326 (1996).

${ }^{26}$ P. Atkins and J. de Paula, Physical Chemistry, 8th ed. (Oxford University Press, London, 2006).

${ }^{27}$ D. Lide, Handbook of Chemistry and Physics, 87th ed. (CRC Press, Boca Raton, 2006).

${ }^{28}$ T. Markussen, R. Rurali, A.-P. Jauho, and M. Brandbyge, Phys. Rev. Lett. 99, 076803 (2007).

${ }^{29}$ J. J. Mortensen, L. B. Hansen, and K. W. Jacobsen, Phys. Rev. B 71, 035109 (2005).

${ }^{30}$ J. Enkovaara et al., J. Phys.: Condens. Matter (to be published).

${ }^{31}$ J. P. Perdew, K. Burke, and M. Ernzerhof, Phys. Rev. Lett. 77, 3865 (1996).

${ }^{32}$ M. Strange, I. S. Kristensen, K. S. Thygesen, and K. W. Jacobsen, J. Chem. Phys. 128, 114714 (2008).

${ }^{33}$ J. M. Soler, E. Artacho, J. D. Gale, A. Garcia, J. Junquera, P. Ordejon, and D. Sanchez-Portal, J. Phys.: Condens. Matter 14, 2745 (2002).

${ }^{34}$ S. Berber and A. Oshiyama, Physica B 376-377, 272 (2006).

${ }^{35}$ S. Berber and A. Oshiyama, Phys. Rev. B 77, 165405 (2008).

${ }^{36}$ R. G. Amorim, A. Fazzio, A. Antonelli, F. D. Novaes, and A. J. R. da Silva, Nano Lett. 7, 2459 (2007).

${ }^{37}$ J. P. Perdew and A. Zunger, Phys. Rev. B 23, 5048 (1981).

${ }^{38}$ P. P. Rushton, Ph.D. thesis, University of Durham, Science Laboratories, U.K., 2002.

${ }^{39}$ M. Moreno, M. T. Barriuso, J. A. Aramburu, P. GarciaFernandez, and J. M. Garcia-Lastra, J. Phys.: Condens. Matter 18, R315 (2006).

${ }^{40}$ S. B. Fagan, R. Mota, J. R. da Silva, and A. Fazzio, Physica B 340-342, 982 (2003). 